This item was submitted to Loughborough's Research Repository by the author.

Items in Figshare are protected by copyright, with all rights reserved, unless otherwise indicated.

\title{
Alcohol and remembering a hypothetical sexual assault: Can people who were under the influence of alcohol during the event provide accurate testimony?
}

\section{PLEASE CITE THE PUBLISHED VERSION}

http://dx.doi.org/10.1080/09658211.2015.1064536

\section{PUBLISHER}

(C) Taylor \& Francis

\section{VERSION}

AM (Accepted Manuscript)

\section{PUBLISHER STATEMENT}

This work is made available according to the conditions of the Creative Commons Attribution-NonCommercialNoDerivatives 4.0 International (CC BY-NC-ND 4.0) licence. Full details of this licence are available at: https://creativecommons.org/licenses/by-nc-nd/4.0/

\section{LICENCE}

CC BY-NC-ND 4.0

\section{REPOSITORY RECORD}

Flowe, Heather D., Melanie K. Takarangi, Joyce E. Humphries, and Deborah S. Wright. 2019. "Alcohol and Remembering a Hypothetical Sexual Assault: Can People Who Were Under the Influence of Alcohol During the Event Provide Accurate Testimony?". figshare. https://hdl.handle.net/2134/21547. 
Alcohol and remembering a hypothetical sexual assault: Can people who were under the influence of alcohol during the event provide accurate testimony? 
Author Note

This research was supported by awards from the Economic and Social Research Council and Alcohol Research UK. 


\begin{abstract}
We examined the influence of alcohol on remembering an interactive hypothetical sexual assault scenario in the laboratory using a balanced placebo design. Female participants completed a memory test 24 hours and 4 months later. Participants reported less information (i.e., responded 'don't know' more often to questions) if they were under the influence of alcohol during scenario encoding. The accuracy of the information intoxicated participants reported did not differ compared to sober participants, however, suggesting intoxicated participants were effectively monitoring the accuracy of their memory at test. Additionally, peripheral details were remembered less accurately than central details, regardless of intoxication level; and memory accuracy for peripheral details decreased by a larger amount compared to central details across the retention interval. Finally, participants were more accurate if they were told they were drinking alcohol rather than a placebo. We discuss theoretical implications for alcohol myopia and memory regulation, together with applied implications for interviewing intoxicated witnesses.
\end{abstract}

Keywords: Alcohol myopia, sexual assault, memory monitoring and control, hypervigilance, intoxication 
Alcohol and remembering a hypothetical sexual assault: Can victims who were under the influence of alcohol during the offense provide accurate testimony?

Serious violent offenses often involve intoxicated witnesses and victims (Evans, Schreiber Compo, \& Russano, 2009; Palmer, Flowe, Takarangi, \& Humphries, 2013). In sexual assault and rape cases ${ }^{1}$, especially, victims and perpetrators are likely to be under the influence of alcohol (Brecklin \& Ullman, 2002; Mohler-Kuo, Dowdall, Koss \& Wechsler, 2004; Testa, 2002). Importantly, intoxicated sexual assault victims are less likely than their sober counterparts to report the offense to the police (e.g., Clay-Warner \& Burt, 2005; WolitzkyTaylor et al., 2011). However, even among simple rape cases-involving acquaintances, no collateral injury, or no weapon use-that are reported and referred for prosecution, charges are less likely to be issued if the victim was drinking (Beichner \& Spohn, 2012).

Alcohol intoxication raises questions about the accuracy of testimony in the minds of criminal investigators (Cole \& Logan, 2010). Similar concerns can also influence trial outcomes: Jurors (e.g., Evans \& Schreiber Compo, 2010) and eyewitness experts (Kassin, Tubb, Hosch, \& Memon, 2001) agree that intoxicated witnesses suffer memory impairment. But, can intoxicated people provide accurate information in legal settings? In the present study, we focused on alcohol and memory impairment in the sexual assault context. Our aims were twofold: we tested whether being under the influence of alcohol, or believing that one has consumed alcohol, would (1) differentially affect the quantity of information women reported about a simulated sexual assault, and (2) interact with other factors to influence the accuracy of women's memory for the sexual assault. In particular, we examined the type of information-central or peripheral to the crime-that women remembered, and the accuracy of their memory over 24 hour and 4 month retention 
intervals following the simulated sexual assault. Although men are sexually assaulted, women as a group are disproportionately affected (Fisher, Cullen, \& Turner, 2000). Owing to this, and the resource limitations we faced, we tested only women.

During a police interview, the interviewee determines what information to volunteer to the police. A crucial question for law enforcement is whether people take into account what their mental state was during the crime when they provide testimony. In the current case, we wondered: would intoxicated participants try to compensate for alcohol-related memory lapses by reporting information only when they are certain that it is accurate? In non-alcohol research, the data show that providing an explicit 'don't know' option increases the accuracy of the information reported (Weber \& Perfect, 2012). Presumably, this option prompts people to monitor and control their memory reports, leading them to report information only when they are certain of its accuracy. Therefore, although people volunteer less information overall, the information they report is more accurate than if they had tried to provide an answer to all of the questions, a pattern of responding known as the informativeness-accuracy trade-off (Brewer \& Weber, 2008; see Koriat \& Goldsmith, 1996).

Research on how people monitor and control their memory reports about an event they witnessed while under the influence of alcohol is limited. In verbal learning research, intoxicated participants respond more conservatively at test than placebo participants (Curran \& Hildebrandt, 1999; Maylor \& Rabbitt, 1987; Mintzer \& Griffiths, 2001; Minzer \& Griffiths, 2002; Soderlund, Parker, Schwartz, \& Tulving, 2005). Only two event memory studies to date have examined the effects of alcohol on the regulation of memory reports. In the first study, Schreiber Compo et al. (2011) had participants consume either alcoholic or non-alcoholic beverages while interacting with a bartender in a lab setting. The alcohol 
group had a peak mean breathalyzed alcohol content $(\mathrm{BrAC})$ of $0.08 \%$. Participants who received an alcohol placebo were more likely to say 'don't know' when reporting their memory for the experience compared to those who received alcohol. However, the memory test occurred 15 minutes after the event, when the alcohol group was still intoxicated. Hence, Schreiber Compo et al. proposed that placebo participants were reporting 'don't know' to compensate for the fact that they thought that their memory was inaccurate due to receiving alcohol. Schreiber Compo et al. further theorized that participants who were actually intoxicated did not have the cognitive resources to compensate, and thus, were less likely to report 'I don't know' and were comparatively less accurate overall. In another study, Schreiber Compo et al. (2011) examined whether 'don't know' responses varied in relation to alcohol intoxication (mean $\mathrm{BrAC} 0.08 \%$ versus mean $\mathrm{BrAC} 0.01 \%$ ) when participants recalled what happened during a staged theft; in this study, however, 'don't know responses' did not vary depending on alcohol condition.

The present study sought to further contribute to this literature. Specifically, we tested whether having actually consumed alcohol-or simply thinking that one had consumed alcohol-leads participants to report 'I don't know' more often in the context of a personally experienced traumatic event. If participants trade-off informativeness for accuracy, then women who encode the event whilst intoxicated may report less information-but be just as accurate in the information they provide-compared to their sober counterparts.

We also prospectively examined whether alcohol affects memory for a hypothetical sexual assault. Generally, laboratory studies-almost all of which employ alcohol doses that result in an estimated $\mathrm{BAC}$ of $0.08 \%$ or less - show that alcohol can have a negative effect on 
episodic memory (e.g., Maylor et al., 1987; Parker et al., 1976; Ray \& Bates, 2006; Hashtoudi et al., 1984; Jones, 1973; Soderlund et al., 2007). However, several studies have not found significant alcohol effects on memory (e.g., Mintzer, Allen \& Griffiths, 2001; Saults, Cowan, Sher, \& Moreno, 2007; Wetherill \& Fromme, 2011; Colflesh \& Wiley, 2013; Knowles \& Duka, 2004; Moulton et al., 2005), so additional research seems warranted. What is more, studies largely involve tests of emotionally neutral verbal learning materials (e.g., word lists). The effects observed may not necessarily generalise to an emotionally negative event such as rape. A few research studies have examined sexual assault victims' retrospective memory reports (e.g., Cooper, Yuille, \& Kennedy, 2002; Cooper, Kennedy, Herve, \& Yuille, 2002; Tromp, Koss, Figueredo, \& Tharan, 1995). However, we can draw only limited inferences from these studies because they are correlational and involve highly select samples. Surprisingly, to our knowledge, only one study has examined how alcohol influences memory for traumatic events (Bisby, Leitz, Morgan, \& Curran, 2010). Using a trauma analogue paradigm, this study found that participants administered a placebo remembered less about horrific traffic accidents compared to participants who had either a mean BrAC of $0.04 \%$ or $0.08 \%$. We sought to extend this work to a sexual assault scenario, using the same alcohol dosage levels and the participant choice procedure (Flowe, Ebbesen \& PutchaBhagavatula, 2007; Flowe, Stewart, Sleath \& Palmer, 2011), which is an interactive testing paradigm that simulates a hypothetical sexual assault. Participants imagined themselves in a hypothetical dating encounter, and self-determined the level of intimate contact that was taking place. Once the participant stopped consenting to the activities being described in the scenario, a hypothetical rape between the participant and the male was described. A particular strength of this paradigm is that it allows for the measurement of consent, which may increase psychological realism compared to scenarios in which participation is passive 
(i.e., where the participant passively reads the scenario, and does not make any decisions about her involvement) (see Flowe et al., 2007). On the other hand, the psychological impact of the scenario, for obvious ethical reasons, of course, is not akin to experiencing an actual rape, and hence, we have to be cautious in generalizing the results from this research to actual rape cases.

We were also interested in whether women would differentially remember information about the hypothetical perpetrator compared to other elements of the scenario depending on alcohol intoxication. According to Alcohol Myopia Theory (AMT), alcohol impairs perception and thought by restricting the focus of attention to central or salient cues to action (Steele \& Josephs, 1990). People are less likely to attend to cues that would inhibit action when they are under the influence. For example, intoxicated people are more likely to engage in unsafe sex when sexually aroused than sober people because arousal leads them to focus on the immediacy and salience of sexual gratification rather than the possible negative repercussions of unprotected sex (MacDonald, Fong, Zanna, \& Martineau, 2000). Applied to eyewitnesses, AMT predicts that eyewitnesses will be more likely to focus on central aspects of the crime, such as the person engaging in criminal behaviour, compared to more peripheral aspects, such as bystanders. As a consequence, alcohol may have a smaller effect on memory for central compared to peripheral information. Several lines of work have tested this prediction.

Schreiber Compo et al. (2011) found that although intoxicated participants were as likely to accurately recall central information (e.g., bartender's hair color) as sober participants, they were less likely to accurately recall subjective and peripheral information about their experience with the bartender (e.g., objects in the surrounding environment, 
such as a dartboard). Participants were tested during the same session; therefore, participants receiving alcohol were intoxicated during both encoding and retrieval. In contrast, Harvey, Kneller and Campbell (2013) found that although intoxicated participants (mean $\mathrm{BrAC} 0.06 \%$ ) were more likely to visually attend to central features of a photo than sober participants (mean BrAC $0.00 \%$ ), their memory for both central and peripheral cues in the photo was no worse than sober participants when tested in a sober state 24 hours later. Other research, focused specifically on identification accuracy, showed that intoxicated and sober participants did not differ in their ability to accurately identify the face of a central character (e.g., perpetrator in a mock crime) when tested either intoxicated (Dysart, Lindsay, MacDonald, \& Wicke, 2002) or sober (Hagsand, Roos-af-Hjelmsäter, Granhag, Fahlke, \& Söderpalm-Gordh, 2013; Yuille \& Tollestrup, 1990). The lack of a difference in identification accuracy across sober and intoxicated participants suggests that alcohol may not affect memory for central information (i.e., the "culprit"). Given the limited number of studies and some conflicting findings, we sought to further investigate whether alcohol would be less likely to affect memory for the perpetrator compared to peripheral aspects of the crime (e.g., bystanders) in the context of sexual assault.

We tested participants' memory 24 hours and 4 months after scenario encoding. We delayed the memory test for a number of reasons: 1 ) sexual assault victims sometimes delay reporting (e.g., Jordan, 2004); 2) the police can decide to postpone interviewing intoxicated victims and witnesses (Evans, Schreiber Compo, \& Russano, 2009); 3) rape typically occurs at night (Office of National Statistics, 2011/12; U.S. Department of Justice, 2008); consequently, in the UK, the victim, after she has had a relatively short initial police interview followed by a physical examination (i.e., "rape kit"), will usually go home to try to 
rest before her memory for the entire event is queried via a lengthy interview. Delayed memory reporting, however, has potentially detrimental effects on memory accuracy, which is known to decrease with increasing delay. This decrease is particularly robust for peripheral compared to central items because memory for central information is more resistant to memory decay (Christianson \& Loftus, 1987). We tested participants again 4 months later because actual victims of sexual assault are interviewed at several stages as their case progresses through the criminal justice system (e.g., police report, trial). By so doing, we could test whether memory reporting and memory accuracy differed after 4 months had passed.

We also varied whether participants expected to consume an alcoholic as opposed to a non-alcoholic beverage. Real world witnesses and victims are typically aware of their alcohol consumption but we wanted to examine whether memory regulation is affected by the belief that one was under the influence of alcohol during scenario encoding. When people believe they are drinking alcohol, they are more likely to engage in social behaviors (e.g., risk-taking, sexual disinhibition) consistent with expectations about how alcohol will make them feel (e.g., relaxed, sociable). On the one hand, basic memory research has found that alcohol expectancy has either small or null effects on cognitive functions such as objective or explicit memory (e.g., memory for word lists) (George \& Marlatt 1986; Hull \& Bond, 1986; Marlatt \& Rohsenow, 1980), possibly because people do not necessarily have pre-existing views about how alcohol affects specific cognitive abilities (Goldman, Brown \& Christiansen 1987; Kvavilashvili \& Ellis 1999). On the other hand, Assefi and Garry (2003) provided evidence that when cognitive tasks have a "social" component, we might expect the same pattern of placebo effects found for social behaviors. Indeed, alcohol expectancy 
effects might even enhance memory for sexual assault. The hypervigilance hypothesis states that women who believe they have consumed alcohol engage in more "hypervigilant" (i.e., cautious) behavior compared to their sober counterparts during scenarios that have the potential to escalate to sexual assault, because drinking alcohol makes them more vulnerable to sexual assault (see Testa et al., 2006). Perhaps this state of hypervigilance during a potentially risky event, in turn, enhances the encoding of the event and improves memory accuracy, a possibility we tested in the current study. Additionally, as discussed above, alcohol expectancy may also influence memory retrieval, with participants who expected alcohol reporting less information about the scenario than their sober participants due to their mental state during scenario encoding.

We tested the following five hypotheses: First, if intoxicated participants take into account their mental state during encoding, they will report 'I don't know" more often than sober participants. Second, intoxicated participants will remember the scenario less accurately overall than their sober counterparts. However, when considering only the questions to which women provided an answer (i.e., the questions to which women did not answer 'I don't know'), accuracy will not differ, thereby demonstrating that intoxicated participants trade-off informativeness for increased accuracy in their memory reports. Third, in accordance with AMT, central information will be remembered more accurately than peripheral information, especially for intoxicated compared to sober women. Fourth, memory accuracy will decrease with increasing delay, especially for peripheral compared to central information. Fifth, women who expected to consume alcohol rather than a nonalcoholic beverage will remember the sexual assault scenario more accurately, as per the hypervigilance hypothesis. 


\section{Method}

\section{Participants}

Eighty-eight women aged $18-31(M=20.42, S D=2.27)$ were enrolled in the study after responding to an advertisement for "female social drinkers" posted at the University of $x$ and passing a number of pre-screenings (described below). Women in the age range of 1624 have a sexual assault victimization rate that is four times higher than other women (Fisher et al., 2000). Nearly all of the women in our sample fell within this age range. Additionally, all of our participants, except for one, were undergraduate students. Relatively high rates of sexual assault victimization are found in undergraduate samples (e.g., Fisher et al., 2000). We did not have any hypotheses regarding memory for the event and women's actual past experiences with sexual assault. Therefore, for ethical reasons, we did not ask participants about their victimization histories because it was unnecessary to remind them about these experiences. Participants received compensation ( $\$ 6$ per hour) for their participation.

Design

We used a 3 (alcohol dose: no alcohol, medium, versus high) x 2 (expectancy: told alcohol versus told tonic) $\times 2$ (information type: central versus peripheral) $\times 2$ (retention interval: 24 hours versus 4 months) mixed design, with alcohol dose and expectancy as the only between participants factors. The dependent variables were information quantity and memory accuracy. Additionally, there were two different types of memory accuracy measures: 1) overall accuracy on the recognition test, and 2) report accuracy, which is the 
accuracy of the information volunteered on the recognition test (i.e., "don't know" responses were excluded in calculating accuracy); please see below for further details.

\section{Materials and Procedure}

The study received ethical approval from the Psychology Research Ethics Committee at the University of $x$. The study was advertised in the campus bulletin and via emails that were sent to the student body. We told participants prior to their enrollment that the study concerned the sexual and dating behaviours of women, and that it may also include discussion of sensitive topics such as rape and sexual assault. This was done for ethical reasons; informing participants about the nature of the study allowed women who wished to avoid discussion of these issues to refrain from enrolling. The overall study procedures consisted of seven phases, all of which were conducted by female researchers and completed individually by the participant.

Phase 1: Online pre-screening. Using an online survey tool, we screened potential participants for problem drinking behaviours on the Alcohol Use Disorders Identification Test (AUDIT), a 10-item questionnaire designed to detect hazardous and harmful alcohol consumption (Saunders, Aasland, Babor, De La Fuente \& Grant, 1993). We also gave participants a general medical questionnaire to identify contraindications for alcohol consumption (i.e. heart or liver disease, psychiatric disorders, pregnancy) and medications known to react negatively with alcohol (e.g., flagyl, prescription pain medications). We invited women to participate in the main experiment if they scored below 11 on the AUDIT, and did not report any alcohol-related contraindications. 
Phase 2: Laboratory Screening. After completing informed consent procedures, participants verified their responses to the pre-screening questionnaires to confirm eligibility. We recorded participants' height and weight measurements, checked photographic identification for proof of age, screened for pregnancy (all participants tested negative, which was required for participation) and verified their breathalyzed alcohol content $(\mathrm{BrAC})$ was $0.00 \%$ at the start of the experiment using the AlcoHawk Slim Digital Alcohol Breath Tester.

Phase 3: Beverage Manipulation. The doses and procedures we used for the beverage manipulation were similar to those widely used in alcohol memory research (e.g., Bisby et al., 2010; Harvey, et al., 2013; McMillen, Smith, \& Wells-Parker, 1989). The researcher prepared the beverages in a separate room. The beverages were prepared to achieve a BrAC level of $.080 \%$ in the high condition and $.040 \%$ in the medium condition, using the formula for women suggested by Curtin and Fairchild (2003), which we calculated separately for each participant using their height and weight information. We chose these BrAC levels to be able to compare the results of our study with the only other study that has examined the effects of alcohol on memory for a traumatic event (i.e., Bisby et al., 2010). Memory accuracy was found to vary across these levels in this research. Additionally, like nearly every single study in the alcohol literature, we did not dose higher than $0.080 \%$ for ethical reasons. We mixed vodka (37.5\%) with tonic water in a 1:5 ratio. Participants assigned to the placebo condition received plain tonic water in a quantity equivalent to the total amount of liquid they would have received in the medium alcohol condition. The researcher divided all beverages into three beakers. 
Following procedures used in other alcohol research (Attwood, Ataya, Benton, Penton-Voak, Munafò (2009), we manipulated alcohol expectancy by clearly labelling every participant's beverage, regardless of whether the participant received alcohol or placebo, as 'Vodka \& Tonic' or 'Tonic Water' and explicitly informing her that her drinks either did or did not contain alcohol. Participants who were told that they had received 'Vodka \& Tonic' were not told anything about the specific dose that they had ostensibly received. We asked participants to consume the drinks within 15 minutes at a pace of 1 drink every five minutes. We breathalyzed participants again 30 minutes after they commenced drinking. Participants were told that the experimenter was following a standard protocol requiring all participants to be treated the same; hence, all participants had to be repeatedly breathalyzed regardless of whether they had actually received alcohol.

Phase 4: Sexual assault Scenario. We used the 'participant choice paradigm' to personally involve participants in a hypothetical "dating" encounter based on actual police cases of sexual assault perpetrated by romantic partners (Flowe et al., 2007; Flowe et al., 2011). As described below, this paradigm enables the participant to control the level of sexual contact taking place between her and a male. The paradigm allows for simulating consent. Typically, researchers ask women to imagine themselves in a rape scenario, which they then read, without making any decisions at all about whether they would elect to be in the situation in the first place, or whether they would consent to any of the sexual contact being described. Previous research has found that allowing participants to self determine consent is critical when investigating variables that affect women's perceptions of rape, and rape reporting (Flowe et al., 2007). 
We presented the scenario in two parts, using E- Prime v 2.0 software. In the first part, participants read introductory information about the male portrayed in the scenario, including his physical appearance, occupation, and possessions. A head and shoulder color photograph of the man accompanied this information. We selected three male perpetrator photographs rated as average in attractiveness from the Radboud Faces database (Langner et al., 2010), and counterbalanced them across participants. We presented the second part of the scenario as 24 sentences, which appeared one at a time on the computer screen. Participants responded to each sentence according to whether or not they wished to remain in the encounter. The first lines of the scenario describe an attractive male behaving in a complimentary way toward the participant. At line 8 of the scenario, consensual foreplay begins (i.e., kissing), and if the participant remains in the scenario, the sexual activity progresses. For women remaining in the scenario until its conclusion, line 24 describes consensual sexual intercourse between the male and the participant. If a participant opts out of the scenario, she is directed to a sub-scenario, which describes a legally definable act of rape. To mitigate any systematic influences on accuracy resulting from the memorability of specific items, we created 16 versions of the scenario, varying the context of the sexual assault (bar, party, his house, participant's house) and the specific details of the scenario (e.g., the man's occupation, the song playing in the background). We randomly allocated participants to scenario version, and recorded the length of time that participants spent reading the scenario. Following the scenario, participants rated how distressing they found the scenario, and how closely they paid attention to it, using an 11-point likert scale, anchored from 1, "not at all" to 11, "extremely". We also asked participants to rate the extent to which they had experienced certain cognitive and physiological effects often associated with alcohol consumption (e.g., "I felt reduced concentration", "I felt dizzy") 
while reading the scenario, using a 7-point likert scale, anchored from 0 , "not at all" to 6, "completely".

Like other research studies, we frequently monitored participants' BrAC (approximately every 30 mins). Participants were not told what their BrAC reading was at any point during the study. Participants were told that they would be released from the study when their BrAC was below $.030 \%$. Participants in the placebo condition were released 2 hours after arriving to the laboratory in order to make it more difficult for them to gauge whether they had received alcohol. On average, participants spent a total of 4 hours in the laboratory.

Phase 5: Memory Test. Scheduling participants to return to the lab 24 hours and 4 months later would have been impossible for us to achieve due to resource limitations and participant availability. We knew that a substantial number of our participants would graduate and move away during the retention interval; therefore, they would be unable to return to the lab 4 months later for further testing. To address this problem, we opted to test participants online. Performance on online cognitive tests is by and large comparable to laboratory tests (e.g., Crump, McDonnell \& Gureckis, 2013). Note that we tested participants online at 24 hours and again at 4 months to hold the testing procedure constant across the two time points.

Twenty-four hours after scenario encoding (and again 4 months later; please see below), we sent participants a survey link, which presented a 30-item recognition test. We opted to use a recognition test because it enabled us to more thoroughly examine participant's memory for the event. Although a recall test is similar to how witnesses are actually questioned by criminal investigators, a recognition format allowed us to 
systematically probe the participant's memory for central versus peripheral information. It was critically important to use a recognition test because there would be no researcher present during the test to probe for additional information. In our pilot work, we have found that follow-up questioning and/or use of a recognition format is critical when asking women to report information about sexual assault, as women can be reluctant to discuss their experience in great detail. We allowed participants to answer 'I don't know' to any given item on the test.

Two key definitions of centrality [relating to emotional events] appear in the literature: thematic centrality (Heuer \& Reisberg, 1990), and visual centrality (Christianson \& Loftus, 1991; see Reisberg \& Heuer, 2004, for a review). We defined central information as information that is related to the perpetrator, because the perpetrator is both thematically and emotionally central to the event. Central cues in this context include the perpetrator's physical appearance, actions, and biographical information (e.g., where he lives and works). We considered other event stimuli (i.e., bystanders and physical surroundings) as peripheral. Twenty-five of our items measured central information (e.g., 'What was Michael's surname? Davies/Jones/Middleton/Smith/I don't know'), and nine items measured peripheral information (e.g., 'What did Michael have to drink? Beer/Rum and Coke/Vodka and Orange/Water' I don't know'). The items are provided in the Appendix.

We also asked participants whether they believed the sexual intercourse that took place during the scenario was consensual, and whether, if the scenario took place in real life, they would report it to the police as rape. Participants responded using an 11-point Likert-type scale, anchored at 1, "definitely no" and 11, "definitely yes". 
Phase 6: Debriefing. Given the sensitive nature of our topic, we arranged for our participants to return to the laboratory for a full debrief. We told them the aim of the study was to investigate reactions to the scenario and how women remembered it. We wanted to debrief participants in person. Because we knew that many of our participants would have moved out of the area by the time that the 4-month memory test was given, we debriefed participants after the first memory test (typically 1-2 weeks later). Participants were not told the alcohol to which condition they had been assigned during the debrief.

Phase 7: Second Memory Test. Participants were contacted 4 months later and asked to complete an online questionnaire regarding the scenario. The questionnaire contained the same 30 recognition questions as the 24 -hour questionnaire. Participants who completed the questionnaire were recompensed with a $£ 3$ online shopping voucher.

\section{Measures and Data Analysis}

We measured information quantity by summing the number of questions the participant answered (i.e., the number of questions to which the participant did not respond with 'I don't know') and dividing it by the total number of test items $(n=30)$. We measured memory accuracy two ways: First, overall test accuracy was determined by summing the total number of answers that the participant got correct and diving the result by the total number of test items. Second, we calculated response accuracy by summing across the total number of items that the participant answered correctly and dividing the sum by the total number of test items that the participant answered.

The dependent variables were analysed using ANOVA and MANOVA. Follow-up tests were conducted using Bonferonni-corrected t-tests. Alpha was set to .05 in all analyses. 
Confidence levels are reported for the means as well as for the effect size estimates, which were computed using $\eta_{p}^{2}$.

Results

Preliminary Results

All of the women who enrolled in the study completed the first recognition test 24 hours later and $73 \%(n=64)$ completed the recognition test again 4 months later. We examined whether the accuracy and quantity of information reported on the first test varied depending on whether participants completed the test 4 months later, and found no significant differences. Among participants who completed the test both 24 hours and 4 months later, $89 \%(n=57)$ experienced the sexual assault scenario (i.e., they withdrew before line 24 , and therefore, they stopped consenting to sexual activity).

We examined whether information quantity and memory accuracy differed in relation to whether women hypothetically consented versus did not consent to sexual intercourse, and no differences were found. We also examined whether information quantity and memory accuracy were related to the point at which women withdrew from the scenario, and no significant associations were found (memory accuracy and withdrawal point: $r=.20, p=.13$; information quantity and withdrawal point: $r=.10, p=.46$ ).

Thus, given that no differences were found between those consented and those who did not, in all subsequent analyses, we analysed data only from women who read the sexual assault scenario and who took the recognition test twice. This allowed us to examine memory for a hypothetical sexual assault at two time points. Note that although the final 
size of the sample ( $n=57$ ) effectively precluded us from being able to examine interactions between dose and expectancy, we did not predict any such effects.

Next, we examined the effects of the dosage procedure on participant BrAC levels to verify that it had worked as expected. Participant BrAC following alcohol administration significantly varied by dose, $F(2,54)=316.59, p<.001, \eta_{p}{ }^{2}=.92\left[C l_{.90}: .88-.94\right]$. Mean $\mathrm{BrAC}$ was significantly lower in the no alcohol condition $\left(M=.00\left[\mathrm{Cl}_{.95}: .00-.00\right]\right)$ compared to the medium and high dose conditions $\left(M=.05\left[C_{.95}: .05-.06\right]\right.$ and $M=.08\left[C l_{.95}: .07-.08\right]$, respectively], and $\mathrm{BrAC}$ in the medium dose condition was significantly lower compared to the high dose condition, $p s<.0001$.

Participants' ratings of their physiological and cognitive state during the experiment appear in Table 1. We entered these measures into a MANOVA, with alcohol dose and expectancy as the independent variables. The pattern of findings obtained across the dependent measures in relation to alcohol dose suggests that women generally felt greater effects consistent with having consumed alcohol as dose increased. Indeed, there was an overall effect of alcohol dose, $F(20,84)=2.00, \eta_{p}{ }^{2}=.32[C l .90: .03-.30]$. Expectancy did not significantly impact any of the measures. We conducted univariate ANOVAs on each of the dependent variables with dose as the independent variable; the results also appear in Table 1. There were significant dose effects for dizziness, increased sociability, blurred vision, slurred speech and reduced concentration.

Dose did not have a significant effect on how emotionally distressing women found the scenario (no alcohol $M=5.67\left[\mathrm{Cl}_{.95}: 4.32-7.02\right]$, moderate $M=6.95\left[C l_{.95}: 5.70-8.20\right]$, high $M=7.06\left[\mathrm{Cl}_{.95}: 5.70-8.41\right]$ ), or how closely they paid attention to it (no alcohol $M=$ $8.28\left[C l_{.95}: 7.38-9.17\right]$, moderate $M=8.38\left[C l_{.95}: 7.55-9.21\right]$, high $M=8.33\left[C l_{.95}: 7.41-\right.$ 
9.23]). These results indicate that women on average tended to find the scenario emotionally distressing and were engaged by it. Additionally, the line at which women withdrew from the scenario did not significantly vary in relation to alcohol dose (no alcohol $M=9.06\left[\mathrm{Cl}_{.95}: 5.88-12.23\right]$, moderate $M=10.48\left[\mathrm{Cl}_{.95}: 7.53-13.42\right]$, high $M=8.72\left[\mathrm{Cl}_{.95}\right.$ : $5.45-11.90])$. The mean values obtained indicate that participants typically withdrew from the scenario about when consensual foreplay began. The length of time that it took for women to read the scenario did not vary depending on dose, suggesting that encoding time was the same on average across conditions. Expectancy condition did not affect emotional distress scores, attention, the line at which women withdrew, or the length of time taken to read the scenario.

Overwhelmingly, women thought that the sexual intercourse was not consensual ( $M$ $=.91\left[\mathrm{Cl}_{.95}: 0.26-1.55\right]$ and said they would report it to the police as rape $(\mathrm{M}=8.57,[\mathrm{Cl} .95$ : $7.99-9.15]$. These results did not vary by alcohol dose or expectancy.

\section{Hypothesis Tests}

Information Quantity. To test Hypothesis 1, which posited that alcohol dose and expectancy would each have an independent effect on the amount of information reported, we conducted a 3 alcohol dose $\times 2$ expectancy $\times 2$ information type $\times 2$ retention interval mixed ANOVA, with information quantity as the dependent variable. As predicted, there was a significant main effect for alcohol dose, $F(2,51)=6.65, \eta_{p}{ }^{2}=.21[C l .90: .04-.34]$. Women in the high condition $\left(M=.56\left[C_{.95}: .49-.63\right]\right)$ reported significantly less information than those in the moderate condition $\left(M=.73\left[\mathrm{Cl}_{.95}: .66-.79\right]\right)$ and the no alcohol condition $\left(M=.70\left[C l_{.95}: .63-.77\right]\right), p^{\prime} s<.05$. Expectancy, however, did not affect the amount of information reported, nor did expectancy and alcohol dose interact, $F(2,58)$ 
$=2.36, p=.10$. Thus, we found partial support for our first hypothesis: women reported less information if they had consumed a relatively high dose of alcohol. No other main effects or interactions were significant.

Memory Accuracy. All of our other hypotheses concerned factors that affect the accuracy of memory. To test these effects, accuracy was submitted to a 3 alcohol dose $x 2$ expectancy $\times 2$ information type $\times 2$ retention interval $\times 2$ accuracy type mixed ANOVA, with information type, retention interval, and accuracy type as within subjects factors. For the variable accuracy type, recall that we scored memory accuracy two different ways; hence, we had two accuracy measures for each participant: overall accuracy, which was calculated based on all 30 test items, where 'I don't know' answers were coded as incorrect, and report accuracy, which was calculated based on the number of test questions to which an answer was volunteered (i.e., 'I don't know' answers were dropped in scoring accuracy).

According to Hypothesis 2, alcohol dose affects overall accuracy (i.e., when accuracy is calculated by treating 'I don't know' answers as incorrect) but not memory report accuracy (i.e., when accuracy is calculated after excluding 'I don't know' answers). A significant main effect was obtained for accuracy type, $F(1,51)=247.94, p<.001, \eta_{p}{ }^{2}=.83$; this effect was qualified by a significant interaction between alcohol dose and accuracy type, $F(2,51)=6.15, p<.01, \eta_{p}{ }^{2}=.19,\left[C l_{.90}: .05-.35\right]$. As illustrated in Figure 1, alcohol dose had a significant effect on overall accuracy, $F(2,51)=4.07, p<.05, \eta_{p}{ }^{2}=.14\left[C l_{.90}: .01-.27\right]$, but no effect on report accuracy. For overall accuracy, women were significantly less accurate in the high condition $\left(M=0.36,\left[\mathrm{Cl}_{.95}: 0.30-0.42\right]\right.$ compared to the no alcohol condition $(M=$ $0.47,\left[\mathrm{Cl}_{.95}: 0.40-0.53\right]$ and compared the medium condition $\left(\mathrm{M}=0.47,\left[\mathrm{Cl}_{.95}: 0.41-0.53\right]\right.$. Thus, the data support Hypothesis 2: when given the opportunity to withhold information 
by reported 'I don't know', the accuracy of information reported by intoxicated women did not differ compared to sober women. These results were not qualified by any of the other study variables.

Like victims and witnesses in other cases, it seems reasonable to assume that women in real world rape cases, determine for themselves the information that they report versus withhold from the police. Thus, in the remaining analyses, report accuracy was the memory measure employed as the dependent variable, and it was entered into a 3 alcohol dose $\times 2$ expectancy $\times 2$ information type $\times 2$ retention interval mixed ANOVA.

We predicted that memory accuracy would be poorer for peripheral compared to central details, especially when women had consumed alcohol (Hypothesis 3). Response accuracy was lower for peripheral information compared to central information, a significant main effect for information type, $F(1,51)=15.88, p<.001, \eta_{p}{ }^{2}=.24\left[C l_{.90}: .08-\right.$ .38]. However, there was no main effect for alcohol dose on response accuracy, and alcohol dose did not significantly interact with information type (see Figure 2). In sum, response accuracy for central details was better than for peripheral details, regardless of alcohol dose. Thus, we found no support for Hypothesis 3.

Hypothesis 4 predicted a larger decrease in accuracy for peripheral compared to central details across the retention interval. Participants were more accurate 24 hours later compared to 4 months after reading the scenario, a main effect for retention interval on report accuracy, $F(1,51)=45.41, p<.001, \eta_{p}{ }^{2}=.47\left[C l_{.90}: .30-.59\right]$. A significant interaction between information type and retention interval qualified these effects, $F(1,51)=21.11, p<$ $.001, \eta_{p}{ }^{2}=.29\left[\mathrm{Cl}_{.90}: .13-.43\right]$. As shown in Figure 3, accuracy decreased across the retention interval for both central and peripheral details $\left(p^{\prime} s<.0125\right)$, and the decrease was larger for 
peripheral compared to central details. Memory accuracy for central compared to peripheral details did not differ at 24 hours (central $M=.75\left[\mathrm{Cl}_{.95}: .71-.78\right]$ versus peripheral $\left.M=.71\left[\mathrm{Cl}_{.95}: .65-.78\right]\right)$, whereas accuracy was significantly greater for central compared to peripheral details at 4 months (central $M=.68\left[\mathrm{Cl}_{.95}: .64-.72\right]$ versus peripheral $\left.M=.46\left[\mathrm{Cl}_{.95}: .38-.54\right]\right)$. Thus, we found support for Hypothesis 4.

Finally, we found a main effect for expectancy, $F(1,51)=4.25, p<.05, \eta_{p}{ }^{2}=.08[C l$. $.90: .00-.21]$. In keeping with Hypothesis 5, the memory reports of women who expected an alcoholic beverage were more accurate overall compared to women who expected a nonalcoholic beverage $\left(M=.69\left[\mathrm{Cl}_{.95}: .64-.74\right]\right.$ versus $\left.M=.61\left[C l_{.95}: .56-.66\right]\right)$. Alcohol dose and expectancy did not significantly interact, $F(2,51)=.39, p=.68$.

In sum, 4 out of 5 of our hypotheses were supported. The results indicated that intoxicated participants were selective about the information that they reported. They reported less information than their sober counterparts (Hypothesis 1), but the accuracy of the information that they reported did not vary in relation to alcohol (Hypothesis 2). Additionally, memory for peripheral information was forgotten over time to a greater extent than peripheral information (Hypothesis 4), and women who expected to consume alcohol were more accurate then those who did expected placebo (Hypothesis 5). Women were more likely to remember central compared to peripheral information regardless of intoxication level, hence Hypothesis 3, which was based on AMT, was not supported.

\section{Exploratory analyses}

The analyses in this section examine how participants' memory reports varied across the retention interval in relation to alcohol dose. We calculated for each participant the 
percentage of information that was 1 ) accurately retained across the interval (hereafter, this variable will be called "information retained"), 2) accurately remembered 24 hours later, but incorrectly remembered 4 months later (hereafter, this variable will be called "information forgotten", 3) inaccurately remembered 24 hours later, but correctly remembered 4 months later (hereafter, this variable will be called "information remembered only at time 2 "), and 4) never correctly remembered at any time point (hereafter, this variable will be called "information never accurately remembered". The results are given in Table 2 as a by alcohol dose and information type. As shown, the rates for information forgotten, and for information remembered only at time 2 were lower, on average, compared to information retained, and information never accurately remembered.

We submitted the measures to two separate MANOVA's, based on Wilks' Lambda, one for central information, one for peripheral information, with alcohol dose as the independent variable in each analysis. Alcohol dose had a effect on remembering central information, but the effect was only marginally significant, $F(10,114)=1.83, p=.06, \eta_{p}^{2}=$ .14. Follow-up univariate ANOVAs indicated that alcohol dose had significant effects on central information never accurately remembered $\left(F(2,61)=4.67, p<.05, \eta_{p}{ }^{2}=.13\right)$, and on central information forgotten $\left(F(2,61)=3.96, p<.05, \eta_{p}{ }^{2}=.11\right)$. As can be seen in Table 3, the average rate for central information never accurately remembered was larger for the alcohol conditions compared to the placebo condition, though, based on Bonferroni corrected post hoc tests, the difference was significant only for the high compared to the placebo condition $(p<.05)$. Additionally, the rate for central information forgotten was larger for women in the placebo condition relative to the alcohol conditions, yet according to Bonferroni corrected post hoc tests, the difference again was significant only for the high 
compared to the placebo condition $(p<.05)$. We surmise this was because sober women encoded more central information to begin with compared to highly intoxicated women, and thus, there was relatively more central information for sober women to forget across the retention interval.

Alcohol dose did not have any significant effects on the variables computed for peripheral information. The descriptive results for this analysis are presented in Table 2.

Finally, we examined the consistency of 'I don't know' responses across the retention interval as a function of alcohol dose and item type. We coded for each item to which the participant answered 'I don't know' at time 1 (i.e., 24 hours), whether at time 2 (i.e., 4 months), the participant also answered 'I don't know', or instead reported a correct answer, or an incorrect answer. We then calculated for each participant for both central and peripheral information three new measures: 1) the percentage of 'I don't know' responses that were consistently answered with 'I don't know" across the retention interval, 2) the percentage of 'I don't know' responses that were later answered correctly at time 2, and 3) the percentage of 'I don't know' responses that were later answered incorrectly at time 2 . The resulting measures were then submitted to two MANOVAs, one for central information and one for peripheral information, with alcohol dose as the independent variable in each analysis. Descriptive statistics for these analyses are reported in Table 3. Alcohol dose did not have a significant effect on the measures, neither for central, nor peripheral items. Table $x$ provides the descriptive results of this analysis. As shown, participants who indicated 'I don't know' at time 1 also tended to report 'I don't know' at time 2, rather than reporting a correct or an incorrect answer. 
Interestingly, the rate of consistent 'I don't know' responses across the interval was larger for central compared to peripheral items, $F(1,59)=6.19, p<.05, \eta_{p}{ }^{2}=.10$. Additionally, women were more likely to provide an incorrect answer at time 2 for central compared to peripheral items to which they had answered 'I don't know' at time $1, F(1,59)$ $=8.35, p<.01, \eta_{p}{ }^{2}=.12$. These results suggest that women were better able to monitor their memories (e.g., know when they do not remember information) across time for central compared to peripheral information. However, this analysis is post hoc in nature, and therefore, caution is warranted in drawing conclusions.

\section{Discussion}

The results from this study contribute to an important applied question: can intoxicated people provide accurate information to police and prosecutors? Our data suggests the answer to this question is yes, at least under the circumstances we studied. Recall that we had two overarching aims. First, we were interested in the effects of alcohol consumption and alcohol expectancy on the quantity of information reported. Second, we were interested in whether the accuracy of information reported about a sexual assault varied depending on alcohol dose, alcohol expectancy and the type of information being recalled (central vs. peripheral). We investigated the effect of these factors on memory accuracy when participants were tested 24 hours and 4 months later.

Alcohol dosage, but not expectancy, influenced the quantity of information participants reported. As dosage increased, the amount of information women reported decreased. Thus, in an applied legal setting, we might expect intoxicated people to report less about their experience than their sober counterparts. But, more importantly, what do our data say about the accuracy of the information intoxicated people report? Overall, the accuracy of 
the information participants reported about the scenario was equally accurate, regardless of whether they were intoxicated or sober. Moreover, central information was remembered more accurately than peripheral information, regardless of alcohol dose. Women's memory for the scenario was worse after 4 months compared to 24 hours, especially for peripheral information; this drop in performance reflects that the forgetting rate was larger for peripheral details compared to information that was central to the sexual assault. Taken together, these findings support the idea that emotionally charged events can be subject to the same basic mechanisms of episodic memory as neutral events (see Rubin, Boals \& Berntsen, 2008).

Based on the AMT framework, we hypothesized that intoxicated participants would be less likely to report and accurately remember peripheral information. AMT posits that greater attentional focus is given to central aspects of an event when people are under the influence of alcohol, which in turn, reduces the amount of attention that is given to more peripheral aspects. Consequently, intoxicated participants should report and accurately remember less peripheral information compared to sober participants. Schrieber Compo et al. (2011) found this pattern, whereby intoxicated participants remembered fewer accurate peripheral details about an interaction they had with a bartender. We did not replicate these findings, however. Instead, we found that participants accurately remembered more central than peripheral details, regardless of intoxication level or expectancy. Two key factors differentiate the present study from Compo et al. (2011): First, we used a negatively charged emotional event, and second, we tested participants after a delay rather than immediately after the crime. The negative emotional nature of the event may have led both intoxicated and sober participants to elaborate on the event during the retention interval, which in turn strengthened memory for the event overall. Additionally, our participants may 
have elaborated on information about the perpetrator to a greater extent than more peripheral aspects of the crime, regardless of dose, and this process may have strengthened memory for central over peripheral information for both sober and intoxicated women (see Christianson, Loftus, Hoffman, \& Loftus, 1991).

Our data in relation to alcohol expectancy suggest that participants may have had particular expectations about how they react under the influence of alcohol and that these expectations changed how they approached the scenario task. Specifically, our data show that women who expected an alcoholic beverage were more accurate than women who expected a non-alcoholic beverage, suggesting that women who believed that they have consumed alcohol were more "hypervigilant" whilst reading the scenario, resulting in better encoding and memory for the event. One could argue, however, that knowledge that one was intoxicated during the event may have affected the way in which information was retrieved from memory during the test. Our data do not support this explanation: women who expected alcohol reported as much information as those who did not expect alcohol. Nevertheless, our results are consistent with the idea that memory tasks with a social component may lend themselves to expectancy effects (Assefi \& Garry, 2003).

Our study contributes to the limited literature that has considered alcohol's influence on memory monitoring and control. In contrast to Schreiber Compo et al. (2011), who found an expectancy effect such that placebo participants were more likely than intoxicated participants to use a 'don't know' option, we found that the likelihood that participants gave a "don't know" response depended on the actual dose of alcohol received, not expectancy. One key methodological difference may explain this discrepancy: our intoxicated participants were tested the next day when they were reportedly sober, whereas Schreiber 
Compo et al.'s intoxicated participants were tested when they were still intoxicated.

Schreiber Compo et al. proposed that intoxicated participants did not have the capacity to regulate their memory reports, due to still being intoxicated at test, and as a result, were less likely to indicate "don't know" compared to placebo participants. Thus, a sober state at test may improve the ability to monitor and control memory reporting. However, we were not in a position to breathalyze participants to confirm that they were sober at test. We have conducted follow-up surveys with participants who have taken part in alcohol research in our lab, and 78\% reported 'yes' when asked if they were sober when they took the online test. Additional research, using procedures that better control the participants mental state at test, are needed to more conclusively test whether participants are better at regulating the accuracy of their memory reports when sober.

There are several avenues for future research we believe important for resolving questions that arise from our data. First, asking participants to freely recall the scenario would align the memory test phase more closely with police practice and with victims' real world experience. In particular, we suggest that using a cognitive interview to measure participants' memory would improve external validity, and allow us to answer additional theoretical and applied questions; for example, issues relating to the influence of question type and misinformation. Second, we know that there are individual differences in participants' tolerance and experience with alcohol consumption, and the effects of that consumption on cognition and behavior while intoxicated (Cox, Yeates \& Regan, 1999; Hiltunen, 1997). But, police do not breathalyze victims and eyewitnesses. Thus, another important practical issue is whether subjective evaluations of intoxication-on the part of the police or of victims - relate to memory accuracy. Third, further research could focus on 
understanding how people initially process and then rehearse central vs. peripheral information. Finally, our data suggest that intoxicated participants may report less information at the memory test relative to their sober counterparts, because they are using a more conservative reporting standard in accordance with their level of intoxication during the scenario. However, we do not know whether participants were actively withholding information they did not feel confident about, or instead saying 'I don't know' because they had no memory for the information. Future research could address this issue by forcing participants to select or report an answer, whilst still giving them the option to withhold that answer by responding "I don't know." This procedure would make it possible to assess what answers participants would have provided in the absence of a "don't know" option (see Weber \& Perfect, 2012). Moreover, it would be interesting to examine what participants believe about how alcohol affects their memory in general, and their confidence in specific answers, both of which may indicate their motivation to withhold information due to perceived alcohol-related memory lapses.

Of course, our study has several limitations. First, similar to other alcohol research (e.g., Conrad, McNamara, \& King, 2012; Mintzer \& Griffiths, 2001), we used a handheld portable breathalyzer, rather than a benchtop model, which would have been more accurate, to estimate BrAC. As such, while we can say that the participants on average were relatively most intoxicated in the high condition, followed by the medium and no alcohol condition, we cannot necessarily draw any inferences about how a specific level of intoxication impacts memory reports. Furthermore, even if we assume that our participants' $\mathrm{BrAC}$ reached a maximum of $0.08 \%$, police may often encounter intoxicated witnesses with a much higher BAC (see Evans, et al., 2009). The overwhelming majority of alcohol studies 
conducted in the lab dose participants to a mean $\mathrm{BrAC}$ of $0.08 \%$ or lower, due to participant safety concerns and attendant insurance considerations raised by University Ethics committees. But, it is also important to consider that the alcohol consumption effects observed in the lab may in fact be larger in the field. Second, as with other research employing analogues to investigate trauma (e.g., Bisby et al., 2010; see Holmes \& Bourne, 2008, for a review), although participants reported that they were emotionally affected by the scenario, our simulated event is, of course, not akin to experiencing a real sexual assault. However, we endeavoured to maximize psychological realism (see Mook 1983) by basing the scenario on real life cases of sexual assault perpetrated by romantic partners and by making the experience as interactive as possible with the participant choice methodology (see Flowe et al., 2007). Third, our data cannot speak to the issue of when intoxicated people should be interviewed, nor do they address whether victims and witnesses should be interviewed while they are still intoxicated versus in a sober state. Fourth, we did not systematically vary whether participants were tested in a sober versus intoxicated state due to resource limitations. We assumed, like the police and other researchers who conduct experiments in the lab and in the field, that most people will be sober when reporting their memory for an event. Fifth, even though our participants were reportedly sober when they completed the recognition measures, they completed the recognition measures online, and hence we could not breathalyze them to verify that they were in fact sober. Thus, we cannot rule out the possibility that state dependency affected memory reports. Nevertheless, state dependency effects tend to be small and idiosyncratic (Duka, Weissenborn, \& Dienes, 2001; Weissenborn \& Duka, 2000) and may affect recall rather than recognition (see Eich, 1980). Additionally, the police also do not breathalyze people when they provide testimony. Sixth, retention interval was measured within subjects, and it is important to note that delay has 
different effects on memory accuracy depending on whether it is measured within or between participants. Previous research implies that our participants would have remembered the event less accurately if their memory had been tested just once four months later as opposed to being tested 24 hours later and again 4 months later (see Ebbesen \& Reinick, 1998). Seventh, our findings are based on a recognition measure of memory, rather than free recall, which may limit the generalizability of our conclusions to legal settings at this time.

To conclude, our findings suggest that intoxicated sexual assault people can provide accurate information to the police, at least within the circumstances that we studied (e.g., 24 hour to 4 month delay, estimated $\mathrm{BrAC}$ of $0.08 \%$ or less). Although compared to sober people, intoxicated people may remember less information, our results imply that when intoxicated people provide testimony, the information they provide is just as accurate as sober people, all other things being equal. Additionally, both sober and intoxicated people alike tend to remember information about the perpetrator over more peripheral information, such as bystanders, and information about the perpetrator is less likely to be forgotten across the retention interval. Information about the perpetrator can provide valuable leads in a sexual assault investigation, thus providing the police with greater opportunity to apprehend sexually violent offenders before they have a chance to victimize other people. 
Table 1. Descriptive (Mean, $\left[\mathrm{Cl}_{.95}\right]$ ) and Inferential Statistics for the Physiological and Cognitive State Measures by Alcohol Dose.

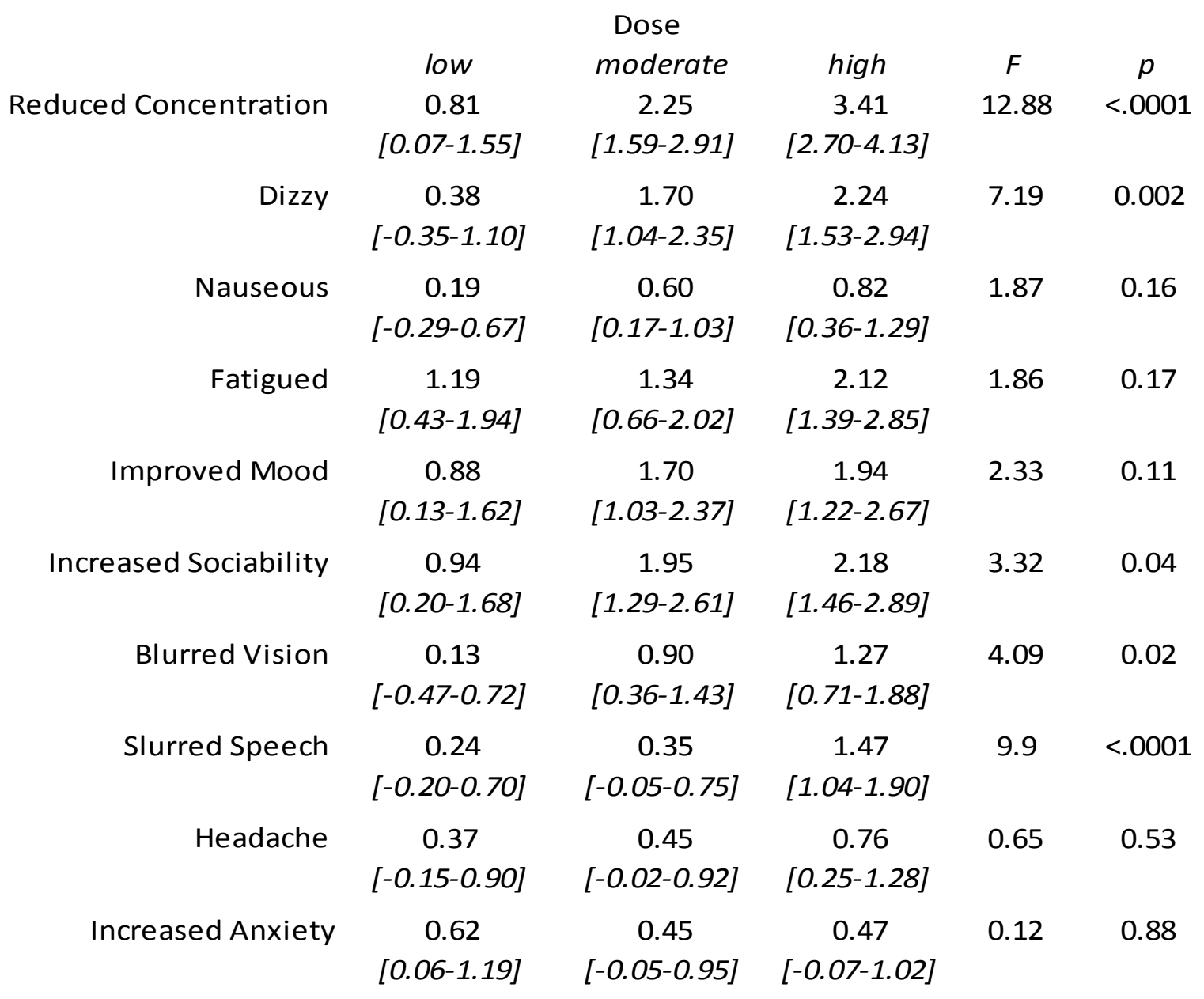


Table 2. Descriptive Statistics Comparing Information (Central and Peripheral Test Items)

Reported at Time 1 (i.e., 24 Hours) and Time 2 (i.e., 4 months).

\section{CENTRAL INFORMATION}

\begin{tabular}{rrrrrr}
\hline & & & & \multicolumn{2}{c}{$95 \% \mathrm{Cl}$} \\
& & & & Lower & Upper \\
& & Mean & SEM & Bound & Bound \\
\hline Information retained & Placebo & 0.36 & 0.03 & 0.30 & 0.41 \\
from time1 to time 2 & Medium & 0.37 & 0.03 & 0.32 & 0.43 \\
& High & 0.31 & 0.02 & 0.26 & 0.35 \\
\hline \multirow{2}{*}{ Information forgotten } & Placebo & 0.24 & 0.02 & 0.20 & 0.28 \\
from time 1 to time 2 & Medium & 0.17 & 0.02 & 0.13 & 0.21 \\
& High & 0.17 & 0.02 & 0.13 & 0.20 \\
\hline remembered accurately & Placebo & 0.09 & 0.02 & 0.06 & 0.12 \\
only at time 2 & High & 0.09 & 0.01 & 0.06 & 0.12 \\
\hline Information never & Placebo & 0.31 & 0.03 & 0.25 & 0.38 \\
accurately remembered & Medium & 0.37 & 0.03 & 0.31 & 0.43 \\
& High & 0.44 & 0.03 & 0.38 & 0.49 \\
\hline
\end{tabular}

PERIPHERAL INFORMATION

\begin{tabular}{rccccc}
\hline & & & & \multicolumn{2}{c}{$95 \% \mathrm{Cl}$} \\
& & & & Lower & Upper \\
& & Mean & SEM & Bound & Bound \\
\hline Information retained & Placebo & 0.28 & 0.04 & 0.19 & 0.36 \\
from time1 to time 2 & Medium & 0.24 & 0.04 & 0.16 & 0.33 \\
& High & 0.18 & 0.04 & 0.10 & 0.25 \\
\hline Information forgotten & Placebo & 0.20 & 0.03 & 0.14 & 0.27 \\
from time 1 to time 2 & High & 0.23 & 0.03 & 0.17 & 0.29 \\
\hline Information & Placebo & 0.06 & 0.02 & 0.02 & 0.10 \\
remembered accurately & Medium & 0.09 & 0.02 & 0.05 & 0.14 \\
only at time 2 & High & 0.06 & 0.02 & 0.02 & 0.09 \\
\hline \multirow{2}{*}{ Information never } & Placebo & 0.46 & 0.05 & 0.36 & 0.56 \\
accurately remembered & Medium & 0.45 & 0.05 & 0.36 & 0.55 \\
& High & 0.53 & 0.04 & 0.44 & 0.62 \\
\hline
\end{tabular}


Table 3. Descriptive Statistics Examining the Consistency of "I don't know" Responses Across the Retention Interval.

\section{CENTRAL INFORMATION}

\begin{tabular}{rrrrcc}
\hline & & & & \multicolumn{2}{c}{$95 \% \mathrm{Cl}$} \\
& & & & $\begin{array}{c}\text { Lower } \\
\text { Bound }\end{array}$ & $\begin{array}{c}\text { Upper } \\
\text { Bound }\end{array}$ \\
\hline Consistently & Placebo & 0.64 & 0.07 & 0.50 & 0.79 \\
answered 'I don't & Medium & 0.66 & 0.07 & 0.52 & 0.80 \\
know' & High & 0.64 & 0.06 & 0.52 & 0.77 \\
\hline Correctly & Placebo & 0.20 & 0.05 & 0.11 & 0.29 \\
answered at time & Medium & 0.14 & 0.04 & 0.05 & 0.22 \\
2 & High & 0.15 & 0.04 & 0.07 & 0.23 \\
\hline Incorrectly & Placebo & 0.16 & 0.05 & 0.05 & 0.26 \\
answered at time & Medium & 0.20 & 0.05 & 0.10 & 0.30 \\
2 & High & 0.21 & 0.05 & 0.11 & 0.30 \\
\hline
\end{tabular}

\section{PERIPHERAL INFORMATION}

\begin{tabular}{rrrrcc}
\hline & & & \multicolumn{2}{c}{$95 \% \mathrm{Cl}$} \\
& & & & Lower & Upper \\
& & Mean & SEM & Bound & Bound \\
\hline Consistently & Placebo & 0.51 & 0.08 & 0.35 & 0.68 \\
answered 'I don't & Medium & 0.51 & 0.08 & 0.34 & 0.68 \\
know' & High & 0.60 & 0.07 & 0.45 & 0.74 \\
\hline Correctly & Placebo & 0.10 & 0.06 & -0.01 & 0.21 \\
answered at time & Medium & 0.19 & 0.06 & 0.08 & 0.31 \\
2 & High & 0.14 & 0.05 & 0.04 & 0.24 \\
\hline Incorrectly & Placebo & 0.39 & 0.08 & 0.23 & 0.54 \\
answered at time & Medium & 0.29 & 0.08 & 0.14 & 0.45 \\
2 & High & 0.26 & 0.07 & 0.13 & 0.39 \\
\hline
\end{tabular}




\section{References}

Assefi, S. L., \& Garry, M. (2003). Absolute memory distortions: Alcohol placebos influence the misinformation effect. Psychological Science, 14(1), 77-80. doi:10.1111/14679280.01422

Attwood, A., Ataya, A., Benton, C., Penton-Voak, I., \& Munafò, M. (2009). Effects of alcohol consumption and alcohol expectancy on the categorisation of perceptual cues of emotional expression. Psychopharmacology, 204(2), 327-334. doi:10.1007/s00213009-1463-1

Beichner, D., \& Spohn, C. (2012). Modeling the effects of victim behavior and moral character on prosecutors' charging decisions in sexual assault cases. Violence and Victims, 27 (1), 3-24. doi:10.1891/0886-6708.27.1.3

Bisby, J. A., Leitz, J. R., Morgan, C. A., \& Curran, H. (2010). Decreases in recollective experience following acute alcohol: A dose-response study. Psychopharmacology, 208 (1), 67-74. doi:10.1007/s00213-009-1709-y

Brecklin, L. R., \& Ullman, S. E. (2002). The roles of victim and offender alcohol use in sexual assaults: Results from the National Violence against Women Survey. Journal of Studies on Alcohol, 63 (1), 57-63. Retrieved from http://www.jsad.com/jsad/article/The Roles of Victim and Offender Alcohol Use in Sexual Assaults Results fr/1319.html

Brewer, N., \& Weber, N. (2008). Eyewitness confidence and latency: Indices of memory processes not just markers of accuracy. Applied Cognitive Psychology, 22 (6), 827840. doi:10.1002/acp.1486

Christianson, S., \& Loftus, E. F. (1987). Memory for traumatic events. Applied Cognitive Psychology, 1(4), 225-239. doi:10.1002/acp.2350010402 
Christianson, S., \& Loftus, E. F. (1991). Remembering emotional events: The fate of detailed information. Cognition and Emotion, 5(2), 81-108. doi:10.1080/02699939108411027

Christianson, S., Loftus, E. F., Hoffman, H., \& Loftus, G. R. (1991). Eye fixations and memory for emotional events. Journal of Experimental Psychology: Learning, Memory, and Cognition, 17(4), 693-701. doi:10.1037/0278-7393.17.4.693

Clay-Warner, J., \& Burt, C. (2005). Rape reporting after reforms: Have times really changed? Violence Against Women, 11 (2), 150-176. doi:10.1177/1077801204271566

Cole, J., \& Logan, T. K. (2010). Interprofessional collaboration on Sexual Assault Response Teams (SART): The role of victim alcohol use and a partner-perpetrator. Journal of Interpersonal Violence, 25 (2), 336-357. doi:10.1177/0886260509334406

Conrad, M., McNamara, P., \& King, A. (2012). Alternative substance paradigm: Effectiveness of beverage blinding and effects on acute alcohol responses. Experimental and Clinical Psychopharmacology, 20(5), 382-389. doi:10.1037/a0029261

Cooper, B. S., Kennedy, M., Hervé, H. F., \& Yuille, J. C. (2002). Weapon focus in sexual assault memories of prostitutes. International Journal of Law and Psychiatry, 25 (2), 181191. doi:10.1016/S0160-2527(01)00107-8

Cooper, B. S., Yuille, J. C., \& Kennedy, M. (2002). Divergent perspectives in prostitutes' autobiographical memories: Trauma and dissociation. Journal of Trauma \& Dissociation, 3 (3), 75-95. doi:10.1300/J229v03n03_05

Cox, W. M., Yeates, G. N., \& Regan, C. M. (1999). Effects of alcohol cues on cognitive processing in heavy and light drinkers. Drug and Alcohol Dependence, 55, 85-89. doi:10.1016/S0376-8716(98)00186-0 
Crump, M. J., McDonnell, J. V., \& Gureckis, T. M. (2013). Evaluating Amazon's Mechanical Turk as a tool for experimental behavioral research. PLoS One, 8(3):e57410. doi: 10.1371/journal.pone.0057410.

Curtin, J. J., \& Fairchild, B. A. (2003). Alcohol and cognitive control: Implications for regulation of behavior during response conflict. Journal of Abnormal Psychology, 112, 424-436. doi:10.1037/0021-843X.112.3.424

Curran, H., \& Hildebrandt, M. (1999). Dissociative effects of alcohol on recollective experience. Consciousness and Cognition: An International Journal, 8(4), 497-509. doi:10.1006/ccog.1999.0417

Dysart, J. E., Lindsay, R. L., MacDonald, T. K., \& Wicke, C. (2002). The intoxicated witness: Effects of alcohol on identification accuracy from showups. Journal of Applied Psychology, 87(1), 170-175. doi:10.1037/0021-9010.87.1.170

Duka, T. T., Weissenborn, R. R., \& Dienes, Z. Z. (2001). State-dependent effects of alcohol on recollective experience, familiarity and awareness on memories. Psychopharmacology, 153(3), 295-306. doi:10.1007/s002130000564

Ebbesen, E. B., \& Rienick, C. B. (1998). Retention interval and eyewitness memory for events and personal identifying attributes. Journal of Applied Psychology, 83(5), 745-762. doi:10.1037/0021-9010.83.5.745

Eich, J. E. (1980). The cue-dependent nature of state-dependent retrieval. Memory \& Cognition, 8(2), 157-173. doi:10.3758/BF03213419

Evans, J., \& Schreiber Compo, N. (2010). Mock jurors' perceptions of identifications made by intoxicated eyewitnesses. Psychology, Crime \& Law, 16 (3), 191-210. doi:10.1080/10683160802612890 
Evans, J. R., Schreiber Compo, N., \& Russano, M. B. (2009). Intoxicated witnesses and suspects: Procedures and prevalence according to law enforcement. Psychology, Public Policy, and Law, 15 (3), 194-221. doi: 10.1037/a0016837

Fisher, B.S., F.T. Cullen, \& Turner, M. G (2000). The Sexual Victimization of College Women. Washington, DC: U.S. Department of Justice, Bureau of Justice Statistics and National Institute of Justice, NCJ 182369.

Flowe, H. D., Ebbesen, E. B., \& Putcha-Bhagavatula, A. (2007). Rape shield laws and sexual behavior evidence: Effects of consent level and women's sexual history on rape allegations. Law and Human Behavior, 31(2), 159-175. doi:10.1007/s10979-0069050-z

Flowe, H. D., Stewart, J., Sleath, E. R., \& Palmer, F. T. (2011). Public house patrons' engagement in hypothetical sexual assault: A test of Alcohol Myopia Theory in a field setting. Aggressive Behavior, 37(6), 547-558. doi:10.1002/ab.20410

George, W. H., \& Marlatt, G. (1986). The effects of alcohol and anger on interest in violence, erotica, and deviance. Journal of Abnormal Psychology, 95(2), 150-158. doi:10.1037/0021-843X.95.2.150

Goldman, M. S., Brown, S. A. \& Christiansen, B. A. (1987). Expectancy theory: thinking about drinking. In H. Blane \& K. Leonard (Eds.), Psychological theories of drinking and alcoholism (pp. 181-226). New York: Guilford Press.

Hagsand, A., Roos-af-Hjelmsäter, E., Granhag, P., Fahlke, C., \& Söderpalm-Gordh, A. (2013). Do sober eyewitnesses outperform alcohol intoxicated eyewitnesses in a lineup? European Journal of Psychology Applied To Legal Context, 5(1), 23-47. 
Harvey, A. J., Kneller, W., \& Campbell, A. C. (2013). The effects of alcohol intoxication on attention and memory for visual scenes. Memory, 21(8), 969-980. doi:10.1080/09658211.2013.770033

Hashtroudi, S., Parker, E. S., DeLisi, L. E., Wyatt, R. J., \& Mutter, S. A. (1984). Intact retention in acute alcohol amnesia. Journal of Experimental Psychology: Learning, Memory, and Cognition, 10(1), 156-163. doi:10.1037/0278-7393.10.1.156

Heuer, F., \& Reisberg, D. (1990). Vivid memories of emotional events: The accuracy of remembered minutiae. Memory and Cognition, 18(5), 496-506. doi:10.3758/BF03198482

Hiltunen, A. J. (1997). Acute alcohol tolerance in cognitive and psychomotor performance: Influence of the alcohol dose and prior alcohol experience. Alcohol, 14, 125-130. doi:10.1016/S0741-8329(96)00115-2

Holmes, E. A., \& Bourne, C. (2008). Inducing and modulating intrusive emotional memories: A review of the trauma film paradigm. Acta Psychologica, 127, 553-566. doi: 10.1016/j.actpsy.2007.11.002

Hull, J. G., \& Bond, C. F. (1986). Social and behavioral consequences of alcohol consumption and expectancy: A meta-analysis. Psychological Bulletin, 99(3), 347-360. doi:10.1037/0033-2909.99.3.347

Jones, B. M. (1973). Memory impairment on the ascending and descending limbs of the blood alcohol curve. Journal of Abnormal Psychology, 82(1), 24-32. doi:10.1037/h0034872

Jordan, J. (2004). Beyond belief? Police, rape and women's credibility. Criminal Justice: International Journal of Policy and Practice, 4(1), 29-59. doi:10.1177/1466802504042222 
Kassin, S. M., Tubb, V. A., Hosch, H. M., \& Memon, A. (2001). On the" general acceptance" of eyewitness testimony research: A new survey of the experts. American Psychologist, 56 (5), 405. doi:10.1037/0003-066X.56.5.405

Koriat, A., \& Goldsmith, M. (1996). Monitoring and control processes in the strategic regulation of memory accuracy. Psychological Review, 103, 490-517. doi:10.1037/0033-295X.103.3.490

Kvavilashvili, L., \& Ellis, J. A. (1999). The effects of positive and negative placebos on human memory performances. Memory, 7(4), 421-437. doi:10.1080/741944922

Langner, O., Dotsch, R., Bijlstra, G., Wigboldus, D. J., Hawk, S. T., \& van Knippenberg, A. (2010). Presentation and validation of the Radboud Faces Database. Cognition and Emotion, 24(8), 1377-1388. doi:10.1080/02699930903485076

MacDonald, T. K., Fong, G. T., Zanna, M. P., \& Martineau, A. M. (2000). Alcohol myopia and condom use: Can alcohol intoxication be associated with more prudent behavior? Journal of Personality and Social Psychology, 78(4), 605-619. doi:10.1037/00223514.78.4.605

Marlatt, A.G., \& Rohsenow, D.J. (1980). Cognitive processes in alcohol use: Expectancy and the balanced placebo design. In N.K. Mello (Eds.), Advances in Substance Abuse (pp.159-199). Greenwich, CN: JAI Press.

Maylor, E. A., \& Rabbitt, P. M. A. (1987). Effect of alcohol on rate of forgetting. Psychopharmacology, 93: 231-235. doi: 10.1007/BF00217069 8295.1993.tb02485.x Maylor, E. A., Rabbitt, P. A., \& Kingstone, A. A. (1987). Effects of alcohol on word categorization and recognition memory. British Journal of Psychology, 78(2), 233. 
McMillen, D., Smith, S., \& Wells-Parker, E. (1989). The effects of alcohol, expectancy, and sensation seeking on driving risk taking. Addictive Behaviors, 14(4), 477-483. doi:10.1016/0306-4603(89)90037-3

Mintzer, M. Z., \& Griffiths, R. R. (2001). Alcohol and false recognition: A dose effect study. Psychopharmacology, 159, 51-57. doi:10.1007/s002130100893

Mintzer, M. Z., \& Griffiths, R. R. (2002). Alcohol and triazolam: Differential effects on memory, psychomotor performance and subjective ratings of effects. Behavioural Pharmacology, 13 (8), 653-658. doi:10.1097/00008877-200212000-00007

Mohler-Kuo, M., Dowdall, G. W., Koss, M. P., \& Wechsler, H. (2004). Correlates of rape while intoxicated in a national sample of college women. Journal of Studies on Alcohol, 65 (1), 37-45. Retrieved from

http://www.jsad.com/isad/article/Correlates of Rape while Intoxicated in a Nati onal Sample of College Women/1107.html

Mook, D. G. (1983). In defense of external invalidity. American Psychologist, 38(4), 379-387. doi:10.1037/0003-066X.38.4.379

Office of National Statistics (2011/12). Focus on Violent Crime \& Sexual Offences. Retrieved from http://www.ons.gov.uk/ons/dcp171778_298904.pdf

Palmer, F. T., Flowe, H. D., Takarangi, M. T., \& Humphries, J. E. (2013). Intoxicated witnesses and suspects: An archival analysis of their involvement in criminal case processing. Law and Human Behavior, 37 (1), 54-59. doi:10.1037/Ihb0000010

Parker, E. S., Birnbaum, I. M., \& Noble, E. P. (1976). Alcohol and memory: Storage and state dependency. Journal of Verbal Learning \& Verbal Behavior, 15(6), 691-702. doi:10.1016/0022-5371(76)90061-X 
Ray, S., \& Bates, M. E. (2006). Acute alcohol effects on repetition priming and word recognition memory with equivalent memory cues. Brain and Cognition, 60(2), 118127. doi:10.1016/j.bandc.2005.07.009

Reisberg, D., \& Heuer, F. (2004). Memory for emotional events. In D. Reisberg \& P. Hertel (Eds.), Memory and Emotion (pp. 3-41). New York: Oxford University Press.

Rubin, D. C., Boals, A., \& Berntsen, D. (2008). Memory in posttraumatic stress disorder: Properties of voluntary and involuntary, traumatic and nontraumatic autobiographical memories in people with and without posttraumatic stress disorder symptoms. Journal of Experimental Psychology: General, 137(4), 591-614. doi:10.1037/a0013165

Saunders, J. B., Aasland, O. G., Babor, T. F., de la Fuente, J. R. \& Grant, M. (1993). Development of the Alcohol Use Disorders Identification Test (AUDIT): WHO collaborative project on early detection of persons with harmful alcohol consumption: II. Addiction, 88 (6), 791-804. doi:10.1111/j.1360-0443.1993.tb02093.x Schreiber Compo, N., Evans, J. R., Carol, R. N., Kemp, D., Villalba, D., Ham, L. S., \& Rose, S. (2011). Alcohol intoxication and memory for events: A snapshot of alcohol myopia in a real-world drinking scenario. Memory, 19 (2), 202-210.

doi:10.1080/09658211.2010.546802

Sexual Offences Act. (2003). Retrieved from http://www.legislation.hmso.gov.uk/acts/acts2003/30042--b.htm.

Söderlund, H., Grady, C. L., Easdon, C., \& Tulving, E. (2007). Acute effects of alcohol on neural correlates of episodic memory encoding. Neuroimage, 35(2), 928-939. doi:10.1016/j.neuroimage.2006.12.024 
Söderlund, H., Parker, E. S., Schwartz, B. L., \& Tulving, E. (2005). Memory encoding and retrieval on the ascending and descending limbs of the blood alcohol concentration curve. Psychopharmacology, 18 (2), 305-317. doi:10.1007/s00213-005-0096-2

Steele, C. M., \& Josephs, R. A. (1990). Alcohol myopia: Its prized and dangerous effects. American Psychologist, 45(8), 921-933. doi:10.1037/0003-066X.45.8.921

Testa, M. (2002). The impact of men's alcohol consumption on perpetration of sexual aggression. Clinical Psychology Review, 22, 1239-1263. doi:10.1016/S02727358(02)00204-0

Testa, M., Fillmore, M. T., Norris, J., Abbey, A., Curtin, J. J., Leonard, K. E., \& ... Hayman, L. r. (2006). Understanding Alcohol Expectancy Effects: Revisiting the Placebo Condition. Alcoholism: Clinical and Experimental Research, 30(2), 339-348. doi:10.1111/j.15300277.2006.00039.x

Tromp, S., Koss, M. P., Figueredo, A., \& Tharan, M. (1995). Are rape memories different? A comparison of rape, other unpleasant, and pleasant memories among employed women. Journal of Traumatic Stress, 8(4), 607-627. doi:10.1002/jts.2490080406

U.S. Department of Justice, (2008). National Crime Victimization Survey. Bureau of Justice Statistics.

Weber, N., \& Perfect, T. J. (2012). Improving eyewitness identification accuracy by screening out those who say they don't know. Law and Human Behavior, 36 (1), 28-36. doi:10.1037/h0093976

Weissenborn, R. R., \& Duka, T. T. (2000). State-dependent effects of alcohol on explicit memory: The role of semantic associations. Psychopharmacology, 149(1), 98-106. doi:10.1007/s002139900349 
Wolitzky-Taylor, K. B., Resnick, H. S., McCauley, J. L., Amstadter, A. B., Kilpatrick, D. G., \& Ruggiero, K. J. (2011). Is reporting of rape on the rise? A comparison of women with reported versus unreported rape experiences in the National Women's StudyReplication. Journal of Interpersonal Violence, 26 (4), 807-832. doi:10.1177/0886260510365869

Yuille, J. C., \& Tollestrup, P. A. (1990). Some effects of alcohol on eyewitness memory. Journal of Applied Psychology, 75(3), 268-273. doi:10.1037/0021-9010.75.3.268 


\section{Footnote}

${ }^{1}$ Sexual assault in the UK is defined as intentionally sexually touching another person without their consent, and rape is defined as intentionally penetrating with a penis another person's vagina, anus or mouth without consent (Sexual Offenses Act, 2003). Heretofore, for the sake of brevity, the term sexual assault will be used when referring to both offenses. 


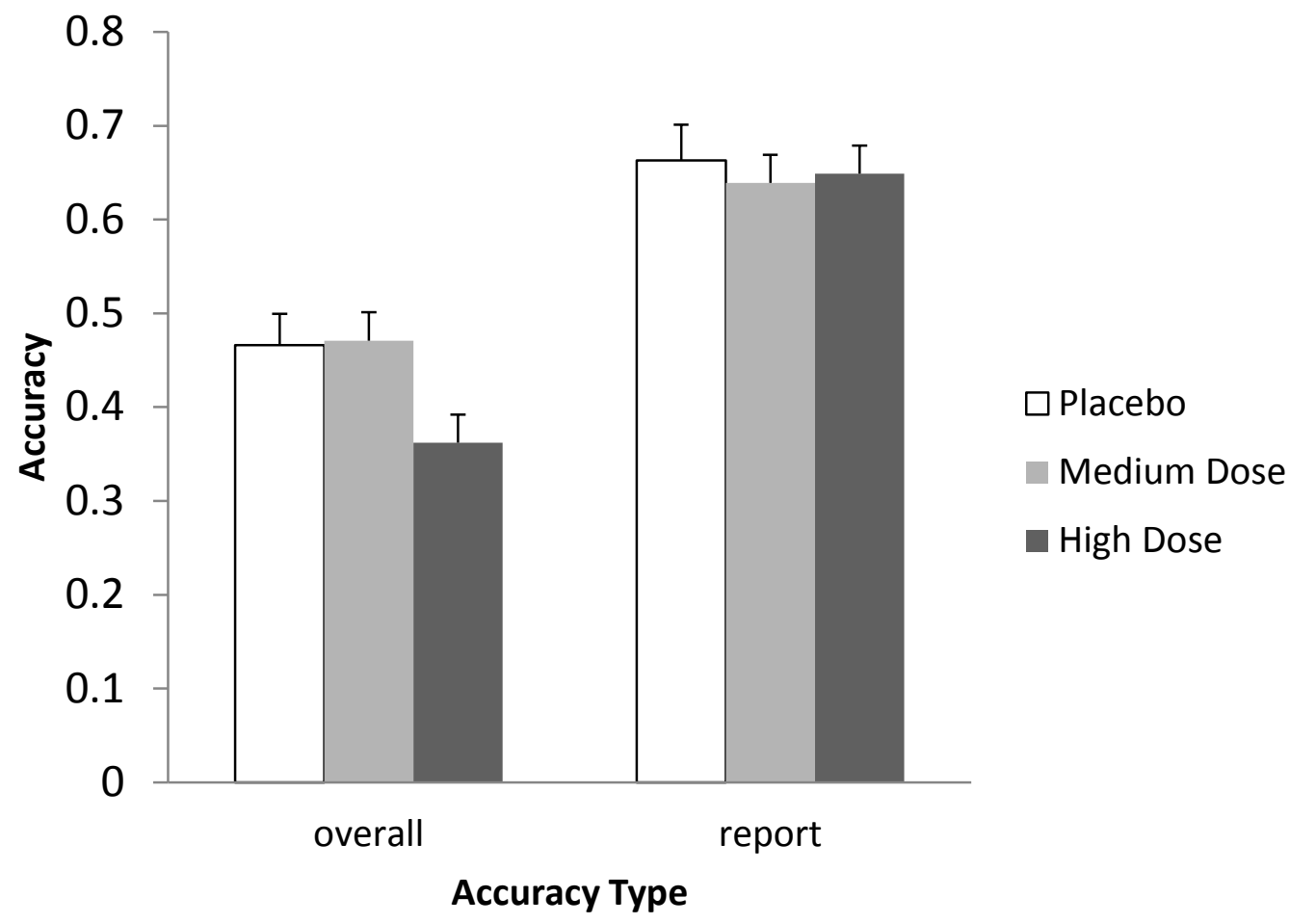

Figure 1. Mean accuracy (+1 SEM) as a function of information type and retention interval. 


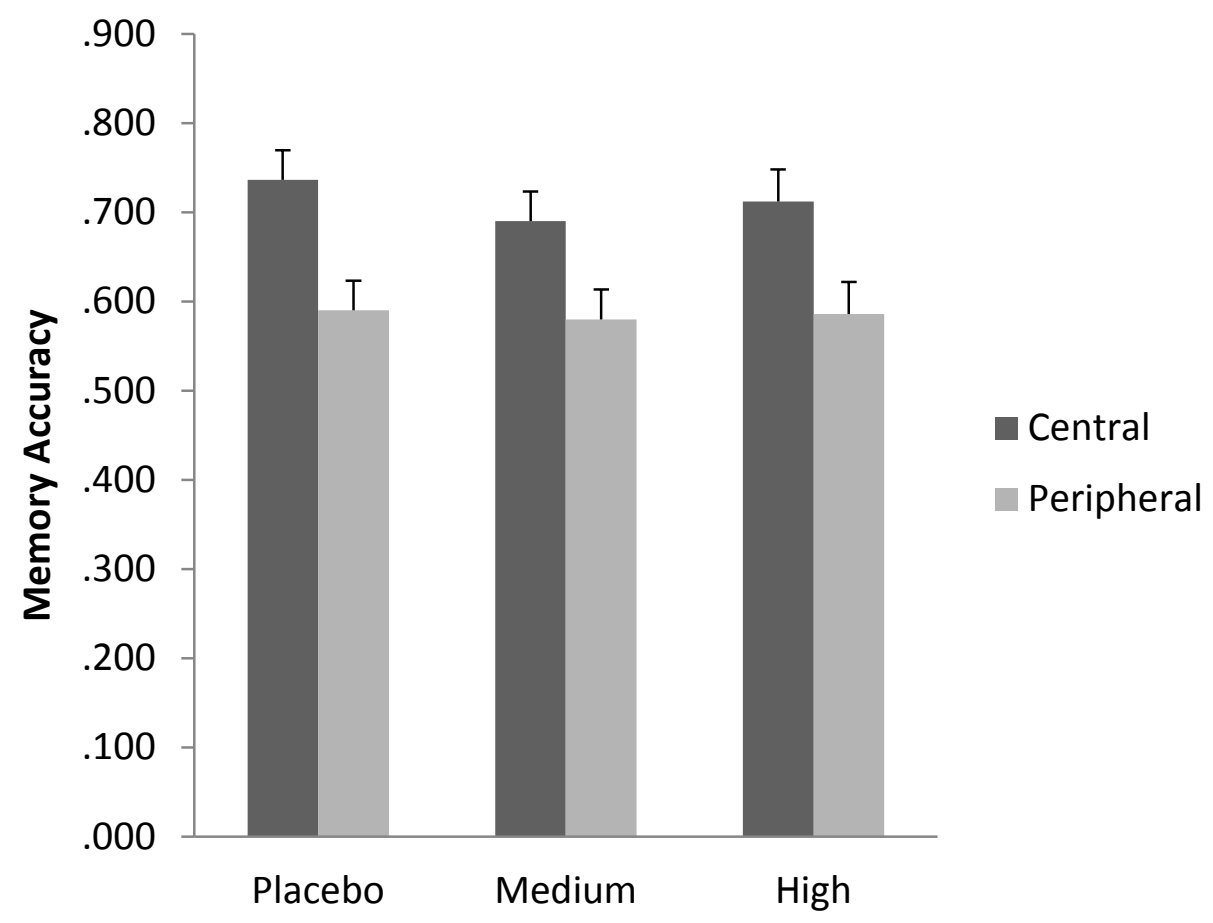

Figure 2. Memory accuracy (+1 SEM) by alcohol dose and detail type. 


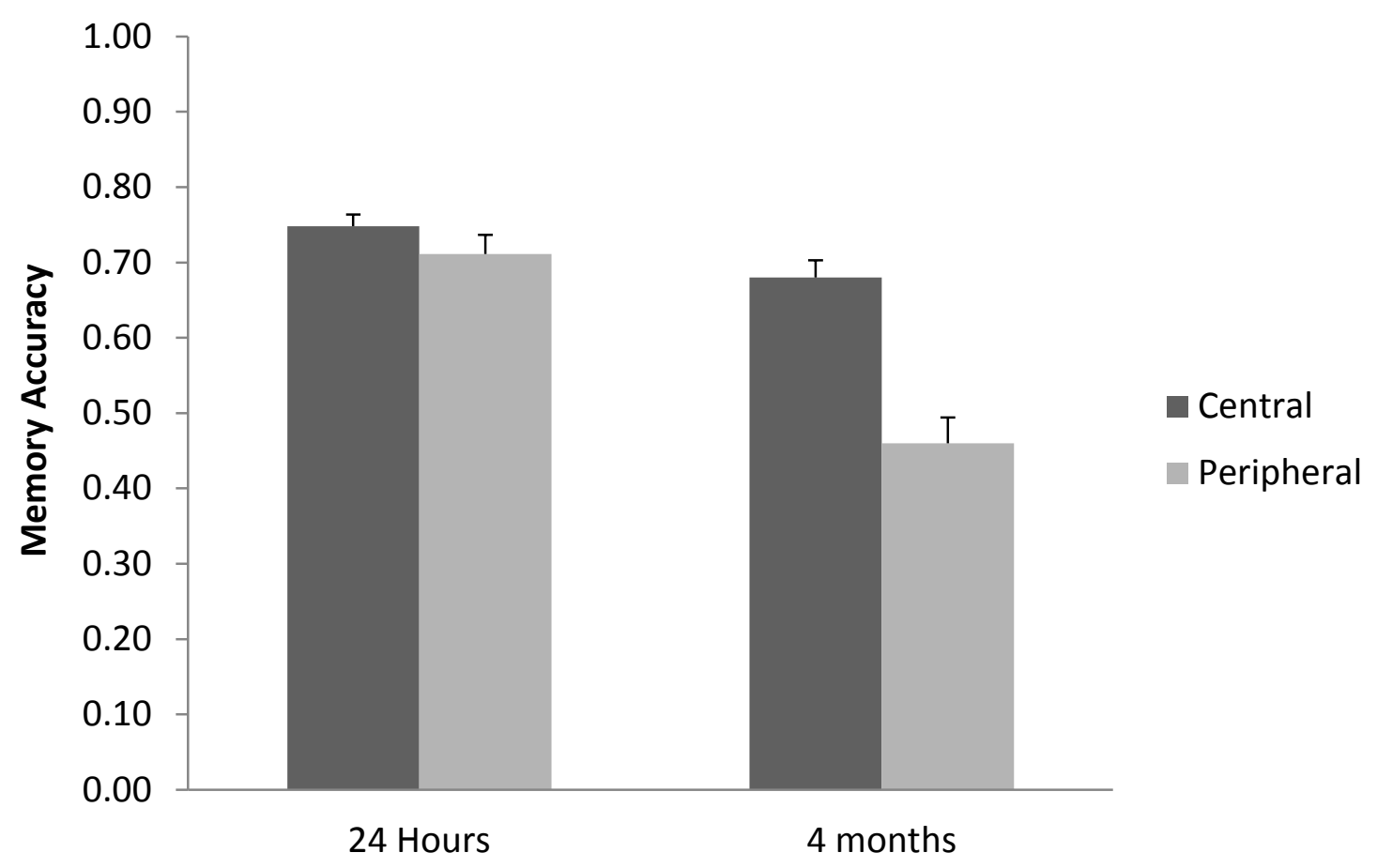

Figure 3. Mean accuracy (+1 SEM) as a function of information type and retention interval. 